Physical Sciences | Farzan Beroz

\title{
Sensing in a disordered environment
}

Dr Farzan Beroz has developed a physical theory of sensing that predicts cell behaviour. Cells measure stiffness by pushing and pulling on their surroundings. However, because their tissue surroundings are disordered, it has been unclear what information cells actually learn by probing. Dr Beroz estimated what a cell learns and predicted that some cells change their shape and move around to gather as much information as possible. Farzan's findings establish a model of cell behaviour and inspire novel ways to engineer high precision sensors.

ells are continuously exposed to mechanical stimuli from (ECM) and their neighbouring cells. These interactions give signals that guide cell behavio from an organism's embryogenesis to its adulthood, including cell differentiation, migration and wound cells probe the stiffness of their

surroundings using mechanosensitive proteins. In response to stiffness activated so that cells adapt and react appropriately. This biological phenomenon is called mechanotransduction.

A small change in mechanical forces can have large implications for cell behaviour. For example, shear stress

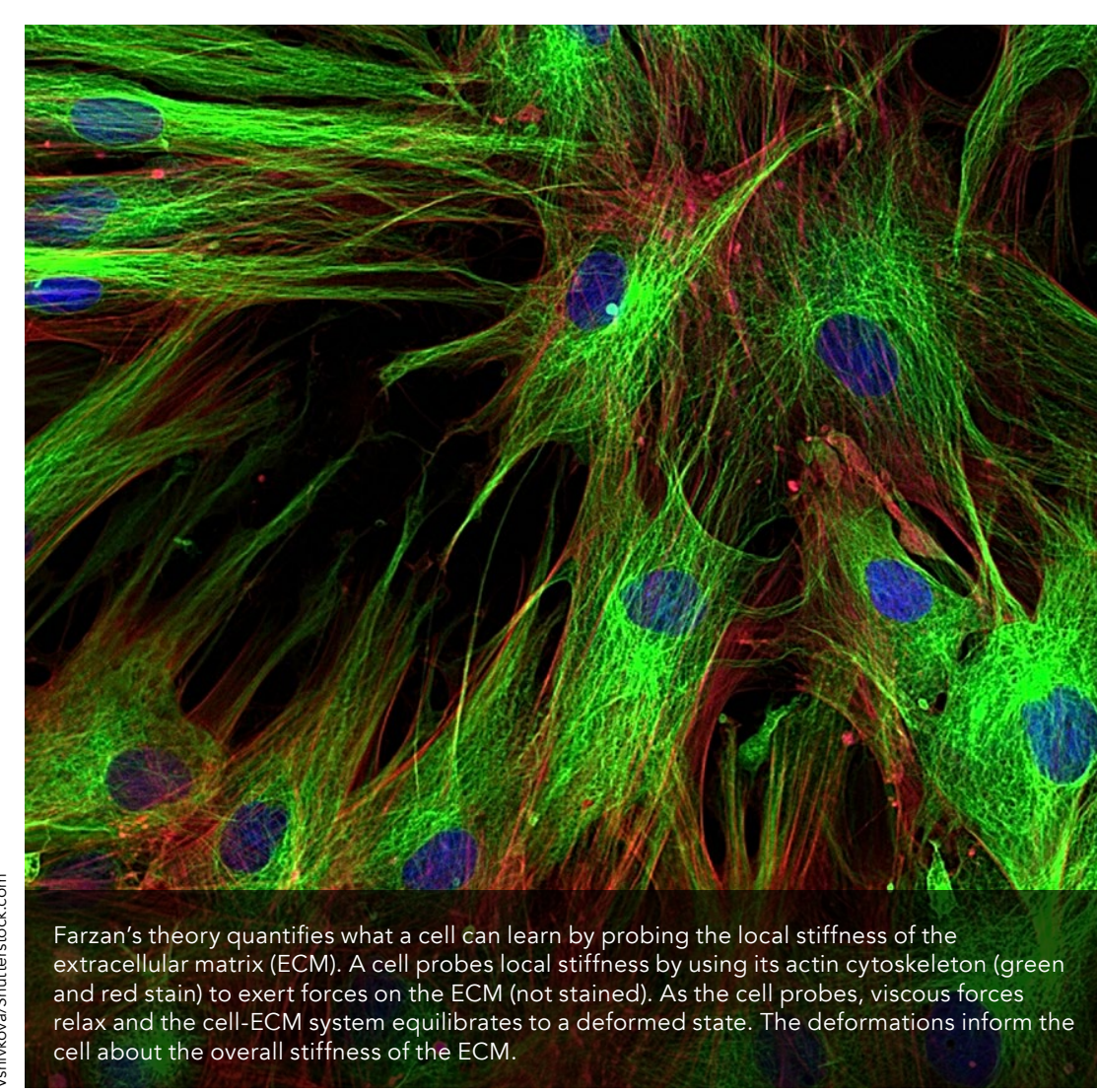

on a developing tissue may determine whether a cell's fate is to become endothelial or hematopoietic. Such decisive changes in behaviour drive metastasis and fibrosis. Thus, mechanical sensing is inextricably linked to wound healing, cancer progression and ageing. To ensure normal bodily function, cells and their microenvironment constantly engage in one another. Mechanosensing entes cells to adapt to changes in their microenvironment.

To add complexity, the cellular microenvironment varies dramatically in composition and topology across different tissues and over time. For example, the ECM is composed of non-cellular components (water, polysaccharides, and proteins includ collagen and fibrin) and provides
scaffolding for cells. It is a highly dynamic structure that cells remode to keep our bodies functional. How do cells sense and gather useful information from a diverse landscape hat shitts over time? Can a cell glean surrounding to keep our bodies running?

\section{INTERSECTING PHYSICS}

\section{AND BIOLOGY}

The relationship between mechanical signals and cell function has become clearer in recent years. Since the start of the millennium, research has identified itracellular molecules that react to cell proniferation, differentiation and migrion. Thanks to advances in mechanobiology experimentation esearchers can now estimate the forces that cells exert to traverse, shape and probe their surroundings. With these exciting new tools, many studies have started to investigate cellular
To date, most cellular mechanosensin studies hos be soft, polyacrylamide gels coated with purified ECM proteins These substrates were engineered to be homogeneous, flat slabs that mimick the overall stiffness of a brain, muscle or bone over their entire surface. The investigations found that cells can actually sense stiffness to distinguish between 2D substrates. In reality, however, connective tissues are highly heterogeneous 3D networks that cells live inside and move through. Culture models also fail to capture the fact that the cellular microenvironment is continually remodelled in living tissue.

To overcome these bottlenecks, mathematical models have been developed to elucidate how cells their tissue surroundings to make important decisions.

Dr Farzan Beroz's childhood fascination with building construction toy models along with his ongoing studies in theoretical physics inspired him to foster an international collaborative effort to understand messy living architectures. He teamed up with researchers from the Max Planck Institute of Dresden, Harvard University, Ludwig-Maximilian University of Munich and Princeton University to discover how the disordered on mechanosensing.

At the time of investigation, researchers had never probed tissue stiffness on the microscale, adding complexity and challenge to this revealing study. Using 3D confocal microscopy images of collagen and fibrin networks, the team developed a computer simulation that accurately captures the heterogeneity and complexity of the ECM. The research team established that in a network environment, different spots exhibit stiffnesses over a wide range. Strikingly, the range is so wide that a single local stiffness measurement does not yield enough information to distinguish tissues as distinct as brain and bone. Thus, rather than optimizing an individual measurement, cells be multiple mocessing the resuls of



Stiffness

Sensory multiplexing could play a decisive role in stem cell differentiation. Schematic illustrations
of how a cell's estimate of tissue stiffness depends sensitively on its microenvironment and on its sensing strategy. Shown here on a logarithmic scale, estimates based on a single force dipole are distributed over a broad range (above), whereas estimates that employ sensory multiplexing are
distributed over a narrow range (below). With a single force dipole, a cell in the brain often feels distributed over a narrow range (below). With a single force dipole, a cell in the brain often feels
spots that are as hard as bone! Taking multiple samples and combining the results in an intelligent
manner may be how a cell decides its fate.

These intelligent strategies exemplify how cells have evolved to cope with an uncertain microenvironment and sense useful information.

Nature Communications in 2017, these findings can explain the presence of hundreds of focal adhesions in some cells. They also imply that cells must use intelligent strategies if they want to sense stifhess accus if they wough to discern tissue identity.

\section{FINDING INTELLIGENT}

\section{STRATEGIES FOR SENSING}

The collaboration's initial findings of

extreme mechanical disorder in tissues that cells cannot discern the identity of their tissue with a single probe, how do cells collect enough information for tissue development and upkeep? This mystery spurred $\mathrm{Dr}$ Beroz to team up with physicists from University of Michigan to develop a set of equations that elucidate the best force a sensor can apply to sense stiffness. For a cell hat is restricted to peforming only one probe, the team proved tha the cells best option is to grab all nearby tissue fbres and pull inwards, a process called 'force dipole'. This is because it feels out the broadest

After a dipolar probe, the cell can collect more information by performing ad the tiss probes to feel out other pants of the tissue. For example, if the cell push and pull simultaneously. Pushing fibres forward and backwards while pulling fibres from the side is a 'force quadrupole'. By pushing and pulling at increasing frequencies, cells glean more bits of information. To collect every last bit, a cell has to perform as he stiffness cues in an intelligent way. This 'sensory multiplexing' strategy ditith predictions, cells appear to use sensory multiplexing to distinguish between substrates that differ in stiffness by as little as $5 \%$. Such precision would enable a cell to distinguish between mechanical properties. extent of the tissue. many distinct probes as it can. The cell completes its inference by averaging 

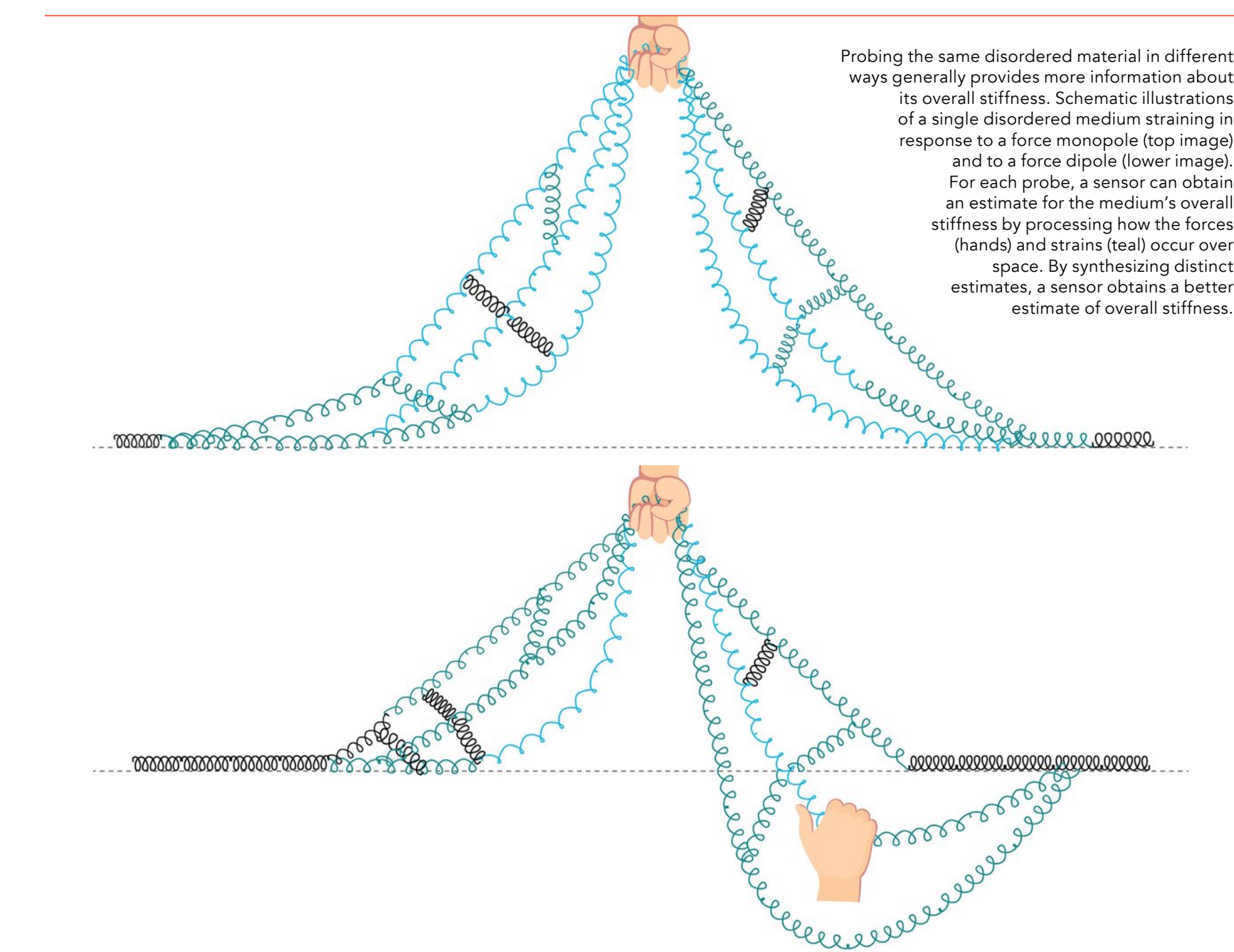

Perspective visualization of a cell probing a surface stiffness are shown by the blue-red colour gradient. Cells attach to their external
environment at discrete protein complexes called
focal adhesions (orange). Here, the surface could correspond to an in vitro gel or to an epithelial
issue These issue. These soft materials end up deformed When cells use their interta
molecular machinery to

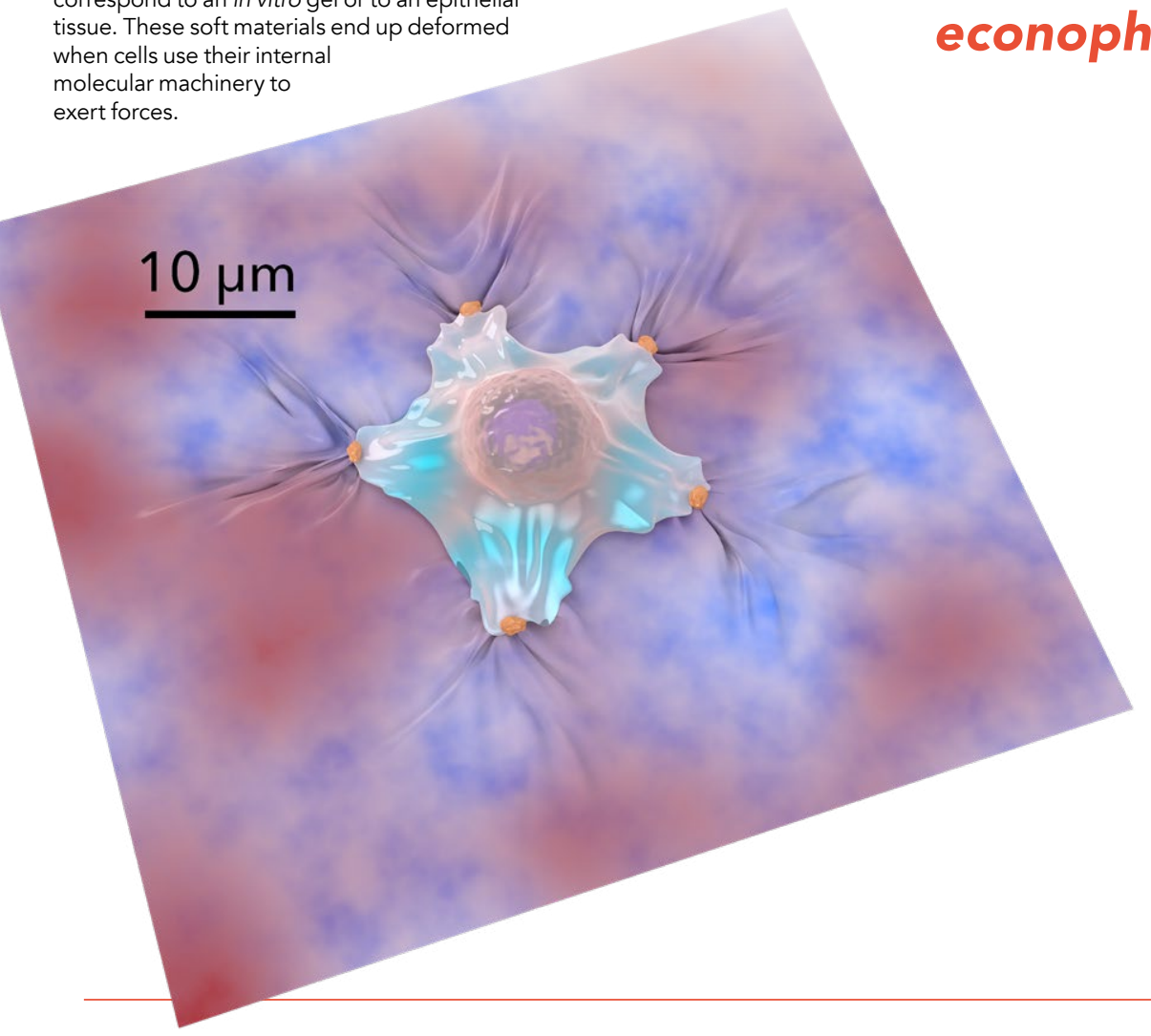

This mathematical model could lead to revolutions in medical sensors, materials science, microrobotics and econophysical modelling.

This study has huge implications for cell biology - the findings can help tumours detect appropriate location to colonise?) and wound healing (how do cells know when to secrete proteins to heal wounds?) Moreover, the study's mathematical framework bears on a remarkably broad range fother systems, including dielectrics, diffusing materials, frictional materials, geomaterials, piezoelectrics, econophysical celcal sensors, microrheology, quality the condexts, the discoveries of Beroz's investigation paveries of Dr making sensors that estime way for properties at the physical limits of spatial resolution. (n)

\section{Dr Farzan Beroz}

E: farzan@umich.edu T: +1 919-744-7138 W: http://www.farzanberoz.com

\section{Research Objectives}

The research of Farzan Beroz aims to uncover the physical principles that shape living architectures,

\section{Detail}

\section{Address}

Farzan Beroz

Church Street

Ann Arbor MI 48109 USA

Bio

Farzan Beroz is a Life Sciences Fellow at the University of Michigan where he develops theories of living architectures. He received his Ph.D. in Physics from Princeton University. Before that, he double majored in Physics and Russian at Duke University.

\section{Funding} Sciences fellowship, the National Science Foundation, Margaret and Herman Sokol Faculty Award, the Harvard Materials Research and Engineering Center, a LewisSigler fellowship, the German Excellence Initiative via the program 'NanoSystems Initiative Manich' (NIM) and .

\section{Collaborators}

Chase P. Broedersz

David K. Lubensky

- Xiaoming Mao

- Stefan Münster

- David A. Weitz

- Ned S. Wingreen

- DiZhou

\section{References}

Beroz, F., Zhou, D., Mao, X. \& Lubensky, D. K. (2020). Physical limits to sensing material properties. Nature Communications, 11, 5170. dol: 10.1038/s41467-020 18995-4

Beroz, F., Jawerth, L. M., Münster, S., Weitz, D. A. et al. (2017). Physical limits to biomechanical sensing in

Doyle, A. D. \& Yamada, K. M. (2016) Mechanosensing via cell matrix adhesions in $3 \mathrm{D}$ micreenvironments. Experimental Cell Research, 343 (1), 60-66. htps.//doi org/10.1016/j.yexcr.2015.10.033

Akhmanova M., Osidak E., Domogatsky S., Rodin S. et al. (2015). Physical, spatial, and molecular aspects of extracellular matrix of in vivo niches and artificial scaffolds relevant to stem cells research. Stem Cells International. https://doi.org/10.1155/2015/167025.

\section{Personal Response} What is the future trajectory of your research in during differentiation?

II We focused on cells embedded inside a medium. geometries and to moving sensors to investigate the trade-offs between undulation and migration. In the long run, I hope to generalise our sensing framework to account for thermal fuctuations, which are relevant cellular trade-offs and thermal fluctuations will be major steps on my path to developing a unified physical
understanding of perception.
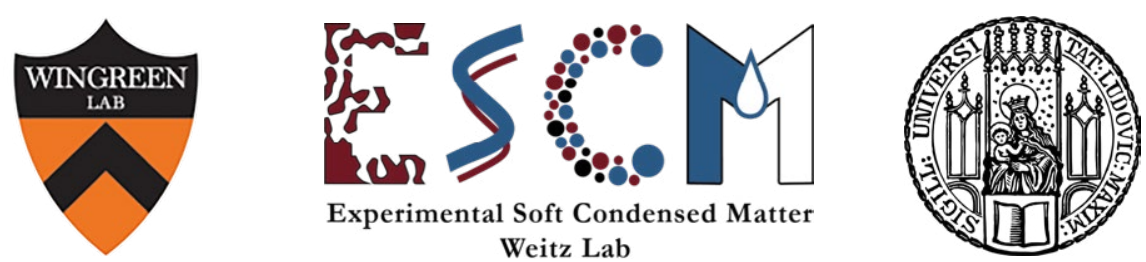